\title{
Novel Design and Research for a High-retaining-force, Bi-directional, Electromagnetic Valve Actuator with Double-layer Permanent Magnets
}

\author{
You Jiaxin ${ }^{1}$, Zhang Kun ${ }^{1}$, Zhu Zhengwei ${ }^{2}$, and Liang Huimin ${ }^{1 *}$ \\ ${ }^{1}$ School of Electrical Engineering and Automation, Harbin Institute of Technology, Harbin 150001, China \\ ${ }^{2}$ Zhenhua Qunying Relay Co., LTD, Guiyang 550018, China
}

(Received 13 September 2015, Received in final form 18 November 2015, Accepted 24 November 2015)

\begin{abstract}
To increase the retaining force, a novel design for a concentric, bi-directional, electromagnetic valve actuator that contains double-layer permanent magnets is presented in this paper. To analyze the retaining-force change caused by the magnets, an equivalent magnetic circuit (EMC) model is established, while the EMC circuit of a double-layer permanent-magnet valve actuator (DLMVA) is also designed. Based on a 3D finite element method (FEM), the calculation model is built for the optimization of the key DLMVA parameters, and the valve-actuator optimization results are adopted for the improvement of the DLMVA design. A prototype actuator is manufactured, and the corresponding test results show that the actuator satisfies the requirements of a high retaining force under a volume limitation; furthermore, the design of the permanent magnets in the DLMVA allow for the attainment of both a high initial output force and a retaining force of more than $100 \mathrm{~N}$.
\end{abstract}

Keywords : magnetic-equivalent-circuit method, electromagnetic valve actuator, permanent magnet, finite element analysis, optimized design

\section{Introduction}

Electromagnetic valve actuators are often used in the pneumatic/hydraulic control system of engines, aeroplanes, and spaceships. The movement and the terminal-position hold of an electromagnetic valve actuator are used for the attainment of a reliable force performance and lower energy consumption. The traditional solenoid actuator uses the electromagnetic force to drive the moveable part during the operational process. For the return process, the spring supplies the mechanical force, which is a process that is due to the technological merits including a simple configuration, a small size, and an easy manufacturing process. Most of the traditional actuators, however, provide only the driving force for one direction and not a latching force; therefore, the traditional solenoid actuator is employed in limited areas. Notably, the actual demand for a bidirectional operation and high latching force is growing [1-3].

For a solenoid actuator containing a mechanical spring, the armature is driven by the electromagnetic force of the

(C)The Korean Magnetics Society. All rights reserved.

*Corresponding author: Tel: +86-0451-86413193

Fax: +86-0451-86413964, e-mail: ibic2013@126.com energizing process and released by the spring force of the de-energizing process [4-5]. Some actuators, however, contain permanent magnets and the corresponding advantages are a low power consumption and high force characteristics in applications. Yet the permanent magnets located in the electromagnetic-flux circuit of the coil may cause a demagnetization problem for a permanent magnet [6-8]. To increase the output force and retaining force for an electromagnetic valve actuator, the available research concerns the design difficulty regarding the actuator in terms of its long moving stroke and a high reliability based on permanent magnets [9-12]. The retaining force represents the force (effect on the armature) under 0 ampere turns that is caused only by the magnets; due to an increasing industry demand, this force has been increased to a high level of approximately $100 \mathrm{~N}$ and the contrary-state retaining force has also been demanded.

This paper is concerned with an electromagnetic valve actuator, the design of which is based on a novel doublelayer, permanent-magnet design, and the aim is the achievement of a maximum energy utility regarding the magnets and maximum retaining force; accordingly, the equivalent magnetic-circuit model and 3D finite-element simulation model are established. Further, the design and optimization of an electromagnetic valve actuator are introduced, 
whereby the prototype actuator is manufactured and a test system is designed. With a focus on force, the experiment results regarding the actuator are presented.

\section{DLMVA Principle and Structure}

\subsection{Structural design based on double-layer perma- nent magnets}

The double-layer permanent magnet valve actuator (DLMVA) consists of a shell, magnets, sleeve, coil, armature, gasket, and cover, as shown in Fig. 1. The coil winding is assembled among the armature that moves in the non-magnetic sleeve and is of a cylindrical construction with two conical terminals. The covers across the axial center of the shell are assembled with an indent that is relative to the armature terminal.

The magnets are $\mathrm{NdFeB}$, and they are produced as a sector-section shape, magnetized in the radial direction, and are installed in two terminals of the coil. Both the design and optimization are discussed under room temperature, but the temperature effect is not considered in this paper [13]. The non-magnetic gasket is used to fill the buffer space, while the magnets that can supply a magnetic retaining force larger than that of the traditional one-layer, permanent-magnet solenoid actuator are located beside the coil; these components are all symmetrical. The shell, cover, and armature of the actuator are composed of the soft magnetic material DT4E.

\subsection{Analysis of the two stable states and the conver-} sion process

The two stable states and the conversion process of the

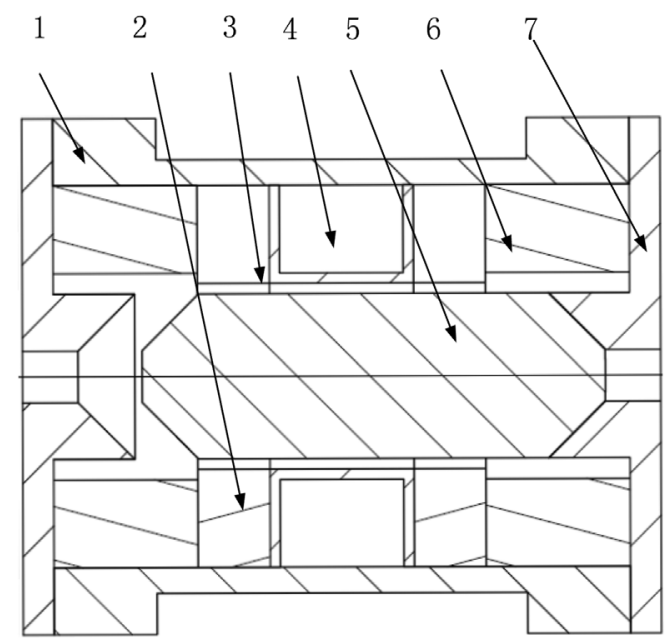

(1) Shell (2) Magnet (3) Sleeve (4) Coil (5) Armature (6) Gasket (7) Cover

Fig. 1. Structure of electromagnetic valve actuator with double-layer permanent magnets.

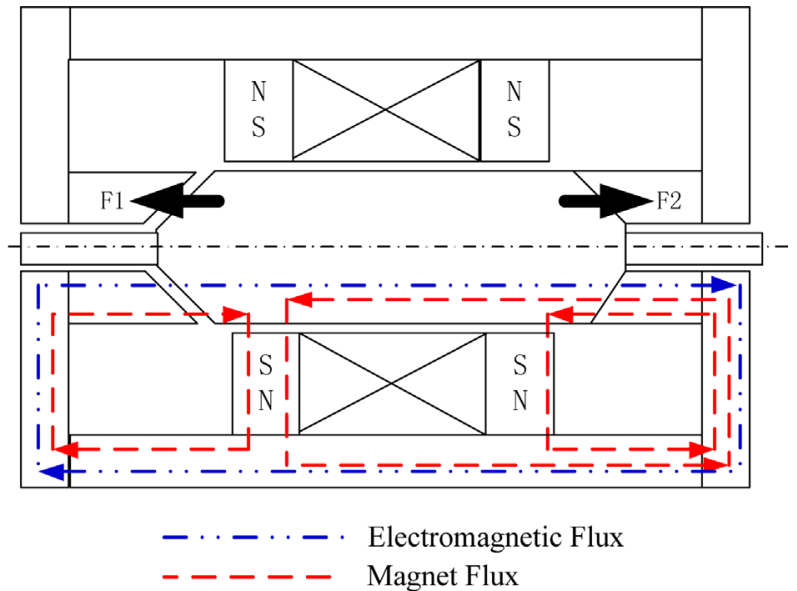

(a) State " 0 " (armature closed at the right side)

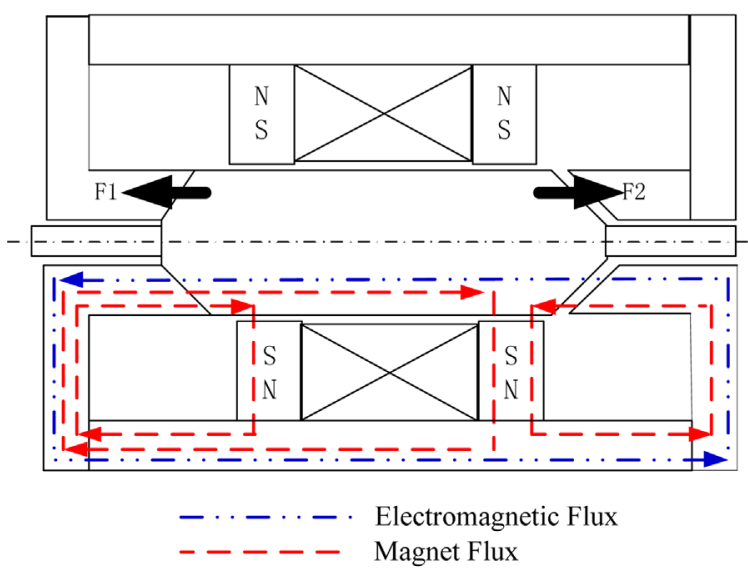

(b) State "1" (armature closed at the left side)

Fig. 2 (Color online) Two stable DLMVA states.

DLMVA are introduced in Fig. 2. In the system, state "0" is defined when the armature is located at the right end, whereas state " 1 " is defined when the armature is located at the left end. The magnetic flux of the magnets is distributed in parallel with the boundary surface among the shell, armature, and cover, and the magnetic-flux density is dependent on the length of the working air gap. When the actuator is in state "0", the armature is driven into the fixed cover by the attractive force that is generated mainly by the down permanent-magnets' flux. By energizing the coil with a positive pulse, the electromagnetic flux increases the attractive force F1 on the topworking air gap; alternatively, the electromagnetic flux decreases the magnets' flux. Meanwhile, F1 and F2 are changing rapidly. When F1 is bigger than F2, the armature is switched to state " 1 ", followed by the movement of the armature to the left. When the coil is energized with a negative pulse, the electromagnetic flux causes F2 to increase and F1 to decrease. When F2 is bigger than F1, 
the armature will be pulled back to state " 0 ".

Because the magnets are not in the electromagnetic-flux circuit, a demagnetization problem regarding the magnet is nonexistent. The energy can be fully utilized, while the force can be adjusted by the type and volume of the magnet. The demand of the higher output force, representing the force under different ampere turns that are caused by the coil and the magnets, can therefore be satisfied.

The state-switching process whereby the armature is closed at two side positions, and for which the small air gap of the magnets produces a maximum retaining force, is described in Fig. 2. Also, the electromagnetic field supplied by the coil is not needed to retain the states " 0 " and " 1 "; therefore, the structural characteristics can achieve a low power consumption and a large retaining force.

\section{General Design and the EMC Model}

\subsection{Basic data of the DLMVA model}

As discussed in part 2, the DLMVA-design parameters, which are within the commonly used volume limitation of $\Phi 48 \mathrm{~mm} \times 60 \mathrm{~mm}$, are listed in Table 1 .

According to these design requirements, the sizes of the parts listed in Table 2 can be obtained.

\subsection{EMC model for DLMVA}

The constructed equivalent magnetic circuit (EMC) model is used to test whether the output force and the retaining force can meet the requirements proposed in this paper.

Neglecting the displacement current and the hysteresis, $F_{\mathrm{m} 1}$ represents the magnetic potential of the top magnets in Fig. 3. Further, $R_{\mathrm{m} 1}$ represents the equivalent magnetic resistance; $R_{11}$ represents the top anti-working, air-gap magnetic resistance; $R_{12}$ and $R_{13}$ represent the top nonworking, air-gap magnetic resistance; $R_{\mathrm{u} 1}, R_{\mathrm{u} 2}, R_{\mathrm{u} 3}, R_{\mathrm{u} 4}$,

Table 1. Basic DLMVA-design requirements.

\begin{tabular}{cc}
\hline \hline Requirement & Design Parameter \\
\hline Volume & $\leq \Phi 48 \mathrm{~mm} \times 60 \mathrm{~mm}$ \\
Displacement & $7 \mathrm{~mm}$ \\
Initial output force & $\geq 20 \mathrm{~N}$ \\
Retaining force & $\geq 100 \mathrm{~N}$ \\
\hline
\end{tabular}

Table 2. Preliminary armature and magnet parameters for DLMVA.

\begin{tabular}{cc}
\hline \hline Size of parts & Measurement \\
\hline Diameter of armature & $16 \mathrm{~mm}$ \\
Height of permanent magnet & $4 \mathrm{~mm}$ \\
\hline
\end{tabular}

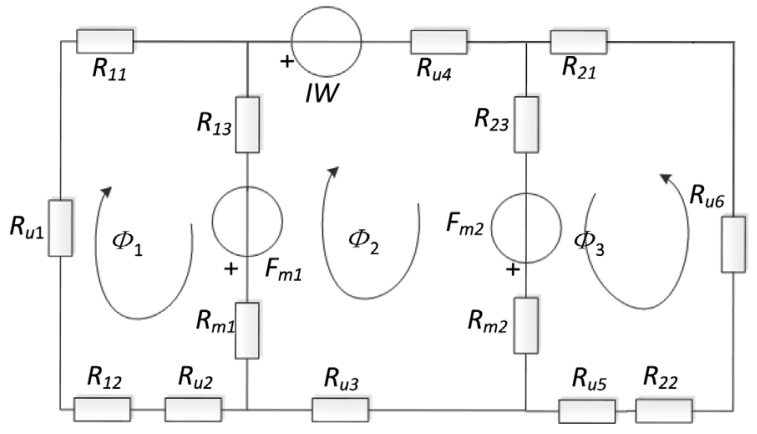

Fig. 3. Electromagnetic equivalent magnetic circuit.

$R_{\mathrm{u} 5}$, and $R_{\mathrm{u} 6}$ represent the resistance of the soft-magneticparts resistance; $F_{\mathrm{m} 2}$ represents the magnetic potential of the bottom magnets; $R_{\mathrm{m} 2}$ represents the equivalent magnetic resistance; $R_{21}$ represents the bottom of the working air-gap magnetic resistance; $R_{22}$ and $R_{23}$ represent the bottom of the non-working air-gap magnetic resistance; $I W$ represents the magnetic potential of the coil; and $\Phi_{1}$, $\Phi_{2}$, and $\Phi_{3}$ represent the circuit flux.

The working air gap and the nonworking air gap $R_{i j}$ are calculated using the following basic function:

$$
R_{i j}=\frac{l_{i j}}{\mu_{0} S_{i j}},
$$

where $l_{i j}$ represents the length of the gap and $S_{i j}$ represents the average area of the gap.

According to the EMC shown in Fig. 3, the following equation is regarding the governing of the static electromagnetic field for the magnetic flux:

$$
\left\{\begin{array}{c}
\left(R_{11}+R_{m 1}+R_{13}+R_{12}+R_{u 2}+R_{u 1}\right) \Phi_{1}-\left(R_{m 1}+R_{13}\right) \Phi_{2}=F_{m 1} \\
-\left(R_{m 1}+R_{13}\right) \Phi_{1}+\left(R_{u 3}+R_{m 1}+R_{13}+R_{u 4}+R_{m 2}+R_{23}\right) \Phi_{2} \\
+\left(R_{m 2}+R_{23}\right) \Phi_{3}=F_{m 2}-F_{m 1}+I W \\
\left(R_{m 2}+R_{23}\right) \Phi_{2}+\left(R_{m 2}+R_{23}+R_{21}+R_{u 5}+R_{22}+R_{u 6}\right) \Phi_{3}=F_{m 2}
\end{array}\right.
$$

The following function is used to set the matrix form:

$$
\boldsymbol{R} \Phi_{b}=F_{b},
$$

where $\boldsymbol{R}$ represents the matrix of the reluctance circuit, $\boldsymbol{F}_{\boldsymbol{b}}$ represents the matrix of the flux circuit, and $\boldsymbol{F}_{\boldsymbol{b}}$ represents the matrix of the magnetic potential-vector circuit.

The magnetic resistance is calculated by the following function:

$$
R=l /(\mu S),
$$

where $S$ represents the average area of the soft magnetic material, $l$ represents the length of the soft magnetic material, and $\mu=B / H$ represents the permeability.

Regarding the nonlinear feature of the soft magnetic material, which is the magnetization curve of the soft 


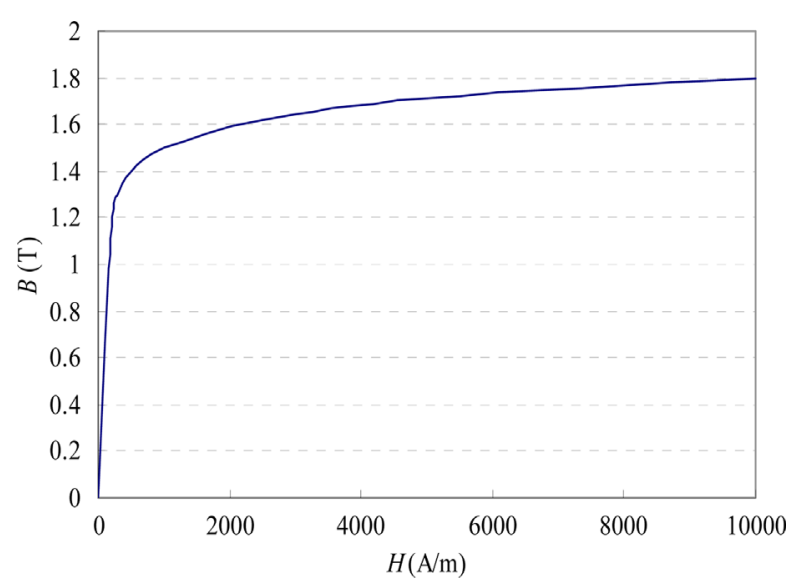

Fig. 4. (Color online) Magnetization curve of the soft magnetic material DT4E.

magnetic material DT4E in Fig. 4, the inner magnetic induction $B$ is smaller than the knee point of the magnetization curve $B_{a}$, and the permeability $\mu$ will increase with the increasing of $B$. The magnetic flux is influenced by the rapid increasing of the magnetic reluctance of the soft magnetic material.

To guarantee accuracy, the permeability $\mu$ is obtained from the test magnetization curve using the Lagrange's interpolation method. When $B_{i-1}<B<B_{i}$, i.e., $B$ is located between $\left(H_{i-1}, B_{i-1}\right)$ and $\left(H_{i}, B_{i}\right)$, the following formula is set:

$$
\mu^{\prime}=\frac{B}{\frac{H_{i}-H_{i-1}}{B_{i}-B_{i-1}}\left(B-B_{i-1}\right)+H_{i-1}} .
$$

Regarding Formula (5), the Newton iteration method is used for the solving process. The variety $\Phi(i)$ is set for the control of the accuracy of the entire model and the verification condition is set as the following formula:

$$
\left|\frac{\Phi(i+1)-\Phi(i)}{\Phi(i)}\right|<\varepsilon
$$

where $\varepsilon$ represents the error-control factor of the model.

Considering the nonlinear characterization of the magnetic material that is according to Formula (1), the working air-gap flux can be calculated using the following iterative loop:

$$
F=\frac{\phi_{2}^{2}}{2 \mu_{0} S},
$$

where $F$ represents the total attraction on the armature, $S$ represents the sectional area of the armature, and $\mu_{0}$ represents the air permeability. The force $F$ can be calculated using Equation (7).
Through the topology analysis, we can obtain the magnetic-flux-circuit equations that can then be used to solve the flux; furthermore, the force can also be calculated. From the elementary calculation results regarding the use of the EMC method, the magnetic resistance and magnetic potential are separated in parallel. The calculation results are $121 \mathrm{~N}$ (state-"1" retaining force) and - $115 \mathrm{~N}$ (state-" 0 " retaining force), showing that the DLMVA can satisfy the design parameters of a $100 \mathrm{~N}$ retaining force in a stable state and an output force of more than $20 \mathrm{~N}$ when the state is transformed from either " 0 " to " 1 " or " 1 " to " 0 " [0 mm, $7 \mathrm{~mm}]$; here, the armature diameter is $16 \mathrm{~mm}$ and the magnet height is $6 \mathrm{~mm}$.

\section{Optimization Design for the Parameters}

The height of the magnet and the space between the armature and the permanent magnets are the two key design parameters that exert an important effect on the retaining force of the actuator. To obtain the optimum values of these two key parameters, an optimization of the parameter design is carried out. First, according to the actuator-structure size, a 3D FEA model is established. Second, the model is meshed with the gap areas using a fine mesh, and the boundary-condition load is set. Lastly, the magnetic-field distribution of the actuator and the force of the armature are calculated.

\subsection{D FEM model for DLMVA}

A 2D FEM model is then built according to the DLMVAstructure size. The magnetic-flux distribution of the center section is shown in Fig. 5. The 2D model is built using a triangle element and the total number of the element is 9179. When the coil is de-energized, the closed loop of the magnetic fluxes passes through the magnets, shell,

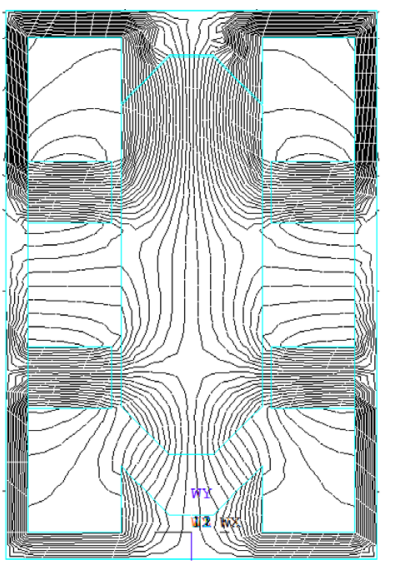

(a) Closed state under 0 ampere turns

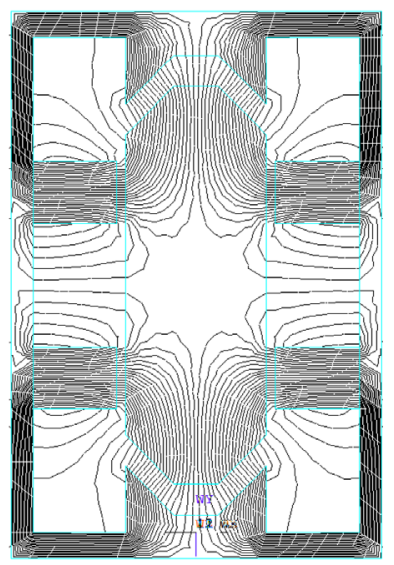

(b) Middle state under 0 ampere turns
Fig. 5. (Color online) 2D FEM model and magnetic-flux distribution in the actuator. 


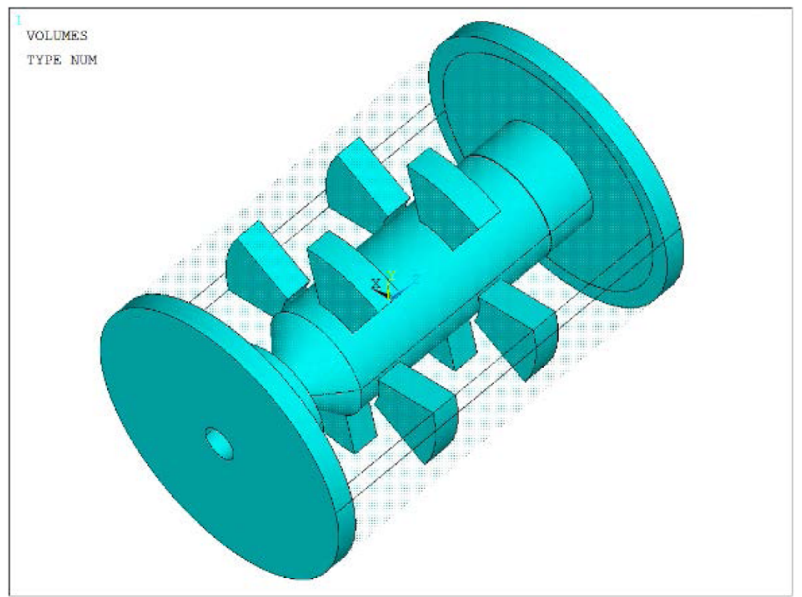

Fig. 6. (Color online) Model of the actuator in ANSYS.

cover, conical air gap, and armature, before it returns to the magnets. The magnetic-flux lines are not vertical to the edge face of the shell or the armature, while the quantity of the flux lines in the armature is in relation to the magnet height and air-gap length that influence the magnetic reluctance over a large force-stroke process; moreover, the performance of the design of the conical air gap is sound in terms of the output force. The improvement of the magnet height, air-gap length, and conical angle for the magnetic actuator are investigated separately, as follows; in terms of defining the distance between the magnets, the ampere turn is locked at approximately 1800 ampere turns.

The optimization for the magnet parameters can be carried out using the 3D FEM model that is established to study the static-force characteristic shown in Fig. 6.

The grid mesh of the model for the magnetic actuator is shown in Fig. 7, whereby a fine mesh in the area of the air gap improves the calculation accuracy. The model

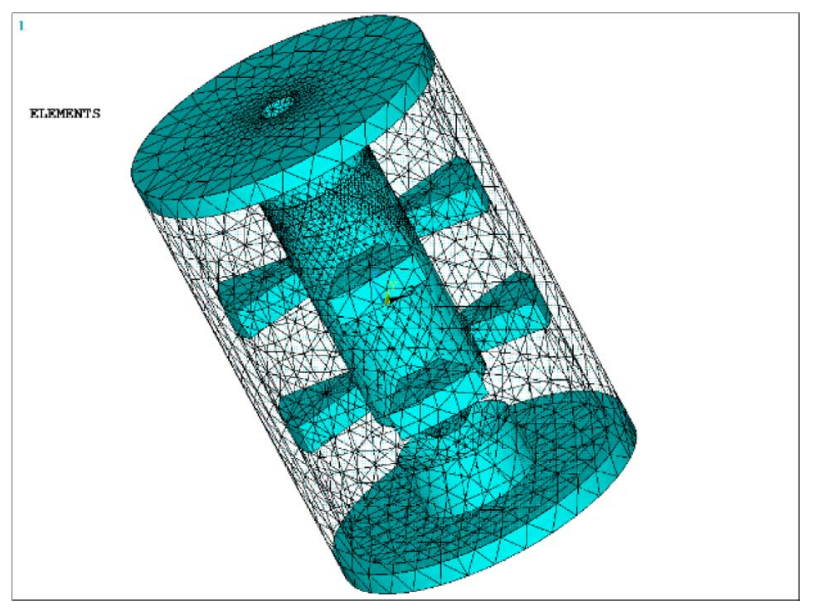

Fig. 7. (Color online) Meshed finite-element model of actuator. contains 14917 elements.

\subsection{Optimization for the magnet parameters based on the 3D FEM}

The stroke-force calculation results under the magnet heights of $5 \mathrm{~mm}, 6 \mathrm{~mm}, 7 \mathrm{~mm}$, and $8 \mathrm{~mm}$ that are based on the 3D FEM are shown in Fig. 7. The four lines on the bottom are the retaining-force curves that correspond to 0 ampere turns. From an analysis of the tendency of the curves when the magnet height is equal to or above 6 $\mathrm{mm}$, the retaining force can be more than $100 \mathrm{~N}$. These top four lines are the output-force curves that correspond to 1800 ampere turns; here, stroke force is defined as the attractive force of the armature and retaining force is defined as the attractive force under 0 ampere turns.

As the magnet height increases, the output and retaining forces are immediately increased. The design of the higher magnets can guarantee the reliability of the motion process; however, the oversized magnets repeatedly increase the volume, weight, and long-term physical damage of the terminal-impact force of the moving armature, and it also increases the output and retaining forces without limitation. So an accurate magnet height of $7 \mathrm{~mm}$ is employed to obtain the suitable stroke-force curve.

\subsection{Optimization for the air gap between the arma- ture and the magnets}

The stroke-versus-force curves under three different airgap lengths of $0.5 \mathrm{~mm}, 1 \mathrm{~mm}$, and $1.5 \mathrm{~mm}$ are shown in Fig. 8 based on the 3D FEM results where the three lines on the bottom are the retaining-force curves. The three lines on the top are the output-force curves under 1800 ampere turns. With the decreasing of the air-gap length,

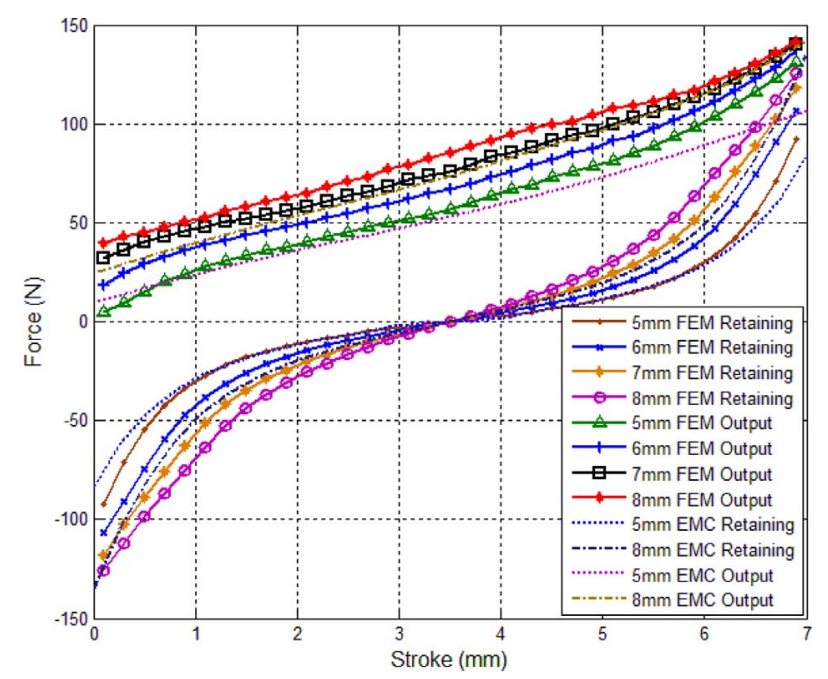

Fig. 8. (Color online) Retaining-force and output-force-characteristics curve under four magnet heights. 


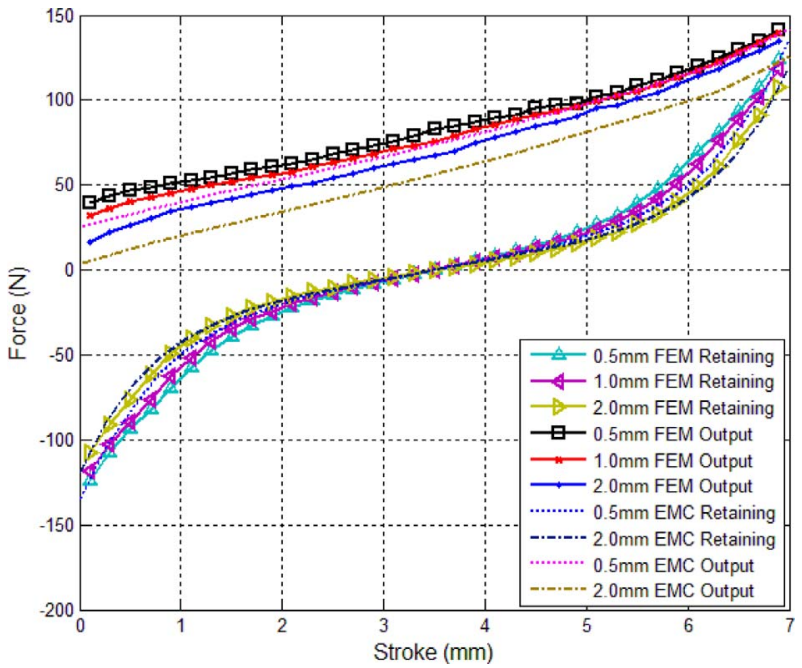

Fig. 9. (Color online) Stroke force under different air-gap lengths.

the force increases and tends to reach a saturated state after a certain value. By analyzing the distribution of the magnetic-flux density, it is possible to detect the point at which the armature is near the saturation status. Considering the volume, weights, total cost demand, and production technology, the optimal space value is $1 \mathrm{~mm}$.

\subsection{Optimization for the angle of the conical arma- ture}

To discuss the effect of the conical air gap on the outputand retaining-force characteristics, the conical angle is designated as a variable value in the model. Figure 10 provides the stroke-force curves under the three conical angles of $40^{\circ}, 45^{\circ}$, and $60^{\circ}$ that are based on the model. As the angle value changes, the curves show the corre-

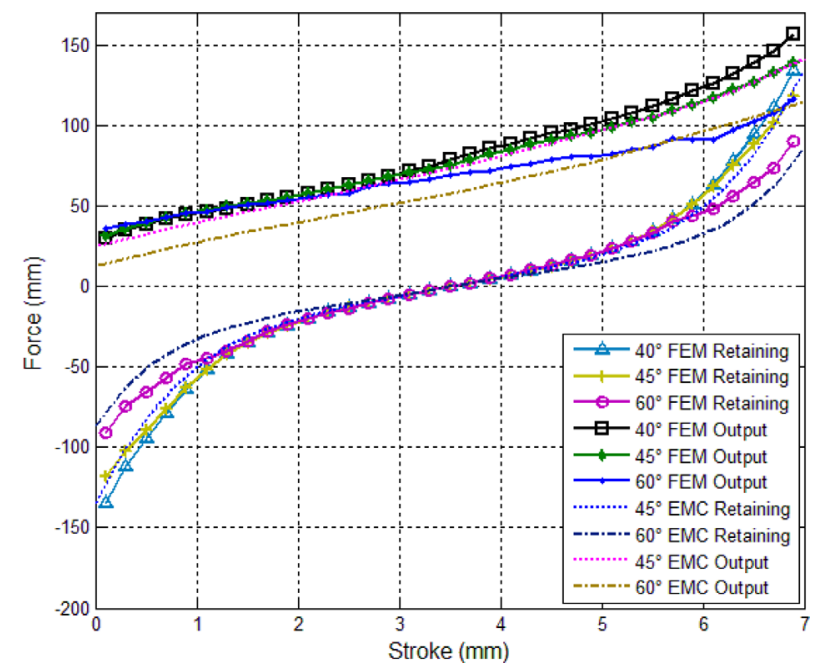

Fig. 10. (Color online) Stroke-force curves under three shell widths. sponding state value, whereby the output-force curves tend to be slightly greater in the initial, a growth drop in the terminal is obvious, and a reduction of the retaining force is distinct. The design of the large conical angle that is used in the traditional cylindrical air gap is therefore unsuitable for a large output force in the terminal, because the mass of the magnetic flux fails to pass the air gap according to the direction of the armature movement, and the armature's contact with the cover is easily lost. The design of the small conical angle is available for the improvement of the retaining force over the large stroke, but it reduces the output force in the initial, and this could lead to an inadequate separation process; therefore, the $45^{\circ}$ parameter can be adopted to balance the conflict between the output force and the retaining force.

\section{DLMVA Prototype and Test}

A DLMVA prototype was manufactured according to the results of the optimization design. The prototype and its test system are shown in Fig. 11.

To test the output force and the retaining force, the force-measurement system including the fixed-device part, the control unit (PLC), and the test equipment (sensor and PC), whereby the PC can guarantee time synchronization, needs to be established first. The accuracy can reach 0.1 $\mathrm{N}$ and the test range is up to $2000 \mathrm{~N}$. The non-magnetic gaskets are manufactured in different sizes so that the force can be tested when the armature is located at a different position. The DLMVA details are shown in

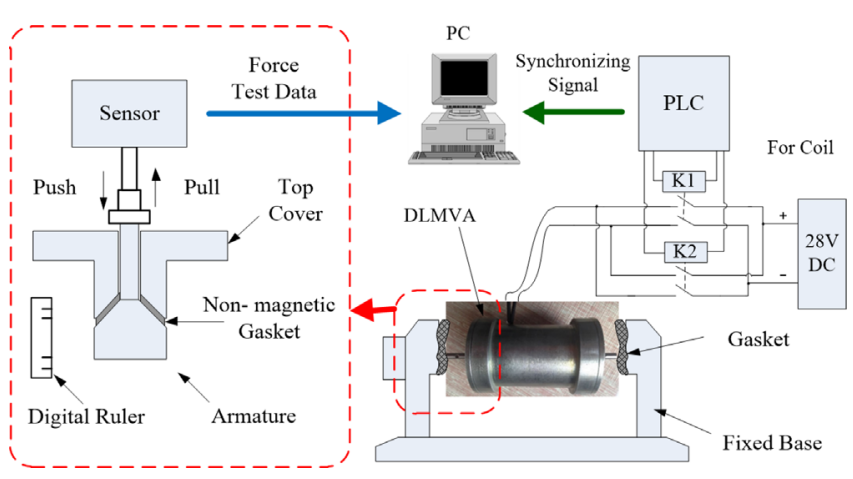

Fig. 11. (Color online) Prototype of DLMVA and test system.

Table 3. Design parameters of DLMVA.

\begin{tabular}{cc}
\hline \hline Design Parameter & Data \\
\hline Magnet height & $7 \mathrm{~mm}$ \\
Armature displacement & $7 \mathrm{~mm}$ \\
Armature conical angle & $45^{\circ}$ \\
Air-gap lengths & $1 \mathrm{~mm}$ \\
Retaining force & $125 \mathrm{~N}$ \\
\hline
\end{tabular}




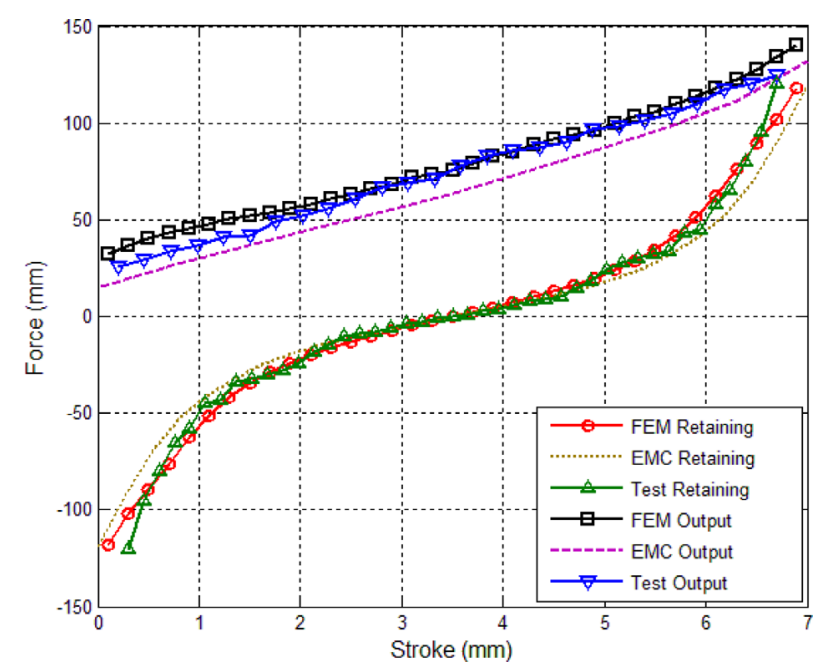

Fig. 12. (Color online) Measured data and simulation data.

Table 3.

The output force of the retaining force and the output force of the armature are tested next. Compared with the magnetic-field-simulation results, the test results are shown in Fig. 12.

The static-force characteristics of the prototype under a rate-coil voltage of $30 \mathrm{~V}$ are shown in Fig. 12. The calculated output-force curve tallies with the trend of the measured output-force curve. Regarding the FEM model, the maximum error is approximately $11.1 \%$ and the average error is $8.6 \%$; regarding the EMC model, the maximum error is approximately $19.1 \%$ and the average error is $12.2 \%$.

The calculated retaining force regarding a $0 \mathrm{~V}$ curve tallies with the trend of the measured retaining-force curve. Regarding the FEM model, the maximum error is $2.3 \%$ and the average error is $1.8 \%$; regarding the EMC model, the maximum error is approximately $16.7 \%$ and the average error is $11.3 \%$. The test results show that the novel DLMVA-actuator structure satisfies the design requirements; in addition, the dynamic characteristic was also tested, producing a very brief making time of $4.7 \mathrm{~ms}$.

\section{Discussion and Conclusions}

1) In this paper, a novel design for an electromagnetic valve actuator that is based on double-layer permanent magnets is presented. The retaining force of the actuator reaches $100 \mathrm{~N}$ through the use of the magnets when the coil is de-energized. The two stable states are converted by a reversed pulse.

2) The EMC model is established for an analysis of the magnetic-circuit topology and a preliminary forecasting of the retaining-force change of the double-layer magnetic circuit. The 2D FEM model is established for an analysis of the flux density. The two key magnet parameters, the height and the air gap, are optimized based on the 3D FEM results.

3) The DLMVA prototype is manufactured according to the optimized design. The test system is built for the measurement of the force. The results of the prototype show that the actuator can satisfy the requirements of output force, energy conservation, and volume size.

Besides, from the single-layer to the double-layer, the design process can lead to a multi-layer design that will increase the force and maintain a rapid manufacturing speed. In terms of a performance improvement, this research may lead to a new perspective regarding magnetcontaining electromagnetic apparatus, and the investigation will be continued in the next research stage.

\section{Acknowledgements}

The authors express their thanks for the support of the NSFC (National Natural Science Foundation of China), Project 51507033, 51177023 and the Heilongjiang Postdoctoral Science Foundation.

\section{References}

[1] Y. Long, J. M, X. Chen, Q. Liang, Y. Shang, and S. Wang, J. Magn. 20, 1 (2015).

[2] Z. Lan and S. Zhuang, J. Magnetic Materials and Devices 2, 68 (2012).

[3] S. Karunanidhia and M. Singaperumalb, Sensors and Actuators A-Physical. 157, 2 (2010).

[4] I. Yatchev, V. Gueorgiev, and K. Hinov, Int. J. Computation and Mathematics in Electrical and Electronic Engineering 28, 5 (2009).

[5] Q. Li, Fan Ding, and C. Wang, IEEE Trans. Magn. 41, 6 (2005).

[6] J. Kim and J. Chang, IEEE Trans. Magn. 43, 4 (2007).

[7] Ji-Young Lee, Ji-Won Kim, and Byung-Chul Woo, J. Magn. 18, 2 (2013).

[8] Y. P. Yang, J. J. Liu, D. H. Ye, Y. R. Chen, and P. H. Lu, IEEE/ASME Trans. on Mechatronics 18, 3 (2013).

[9] Q. Li, F. Ding, and C. Wang, IEEE Trans. Magn. 41, 6 (2005).

[10] J. Lee, E. M. Dede, D. Banerjee, and H. Lizuka, Finite Elements in Analysis and Design 58, (2012).

[11] R. Olaru, C. Astratini-Enache, and C. Petrescu, Int. J. Applied Electromagnetics and Mechanics 38, 2 (2012).

[12] J. B. Wang, K. Atallah, and W. Y. Wang, IEEE Trans. Magn. 47, 10 (2012).

[13] M. Noh, M. J. Gi, D. Kim, Y.-W. Park, J. Lee, and J. Kim, J. Magn. 20, 1 (2015). 